https://helda.helsinki.fi

\title{
Modeling Monty Hall in IF logic
}

\section{Sandu, Gabriel}

Springer International Publishing 2017-03

pÿSandu, G \& Velica, S 2017 , Modeling Monty Hall in IF logic . in C Ba_kent, L S Moss \& R Ramanujam (eds), Rohit Parikh on Logic, Language and Society . Outstanding

Contributions to Logic , vol. 11 , Springer International Publishing , Cham , pp. 165-184 . https://doi.org/10.1007/978

http://hdl.handle.net/10138/312090

https://doi.org/10.1007/978-3-319-47843-2_10

unspecified

acceptedVersion

Downloaded from Helda, University of Helsinki institutional repository.

This is an electronic reprint of the original article.

This reprint may differ from the original in pagination and typographic detail.

Please cite the original version. 


\title{
Modeling Monty Hall in IF Logic
}

\author{
Gabriel Sandu and Silviu Velica
}

\section{Monty Hall: Formulation of the problem}

Let us start by considering two formulations of the Monty Hall problem (Grinstead and Snell, Introduction to Probabilities).

The first formulation is:

Suppose you are on a Monty Hall's Let's Make a Deal!You are given the choice of three doors, behind one door is a car, the others goats. You pick up a door, say 1, Monty Hall opens another door, say 3, which has a goat. Monty says to you "Do you want to pick door 2?" Is it to your advantage to switch your choice of doors? (Grinstead and Snell, Introduction to Probabilities, Example 4.6, p. 136)

The second formulation is more general:

We say that $C$ is using the "stay" strategy if she picks a door, and, if offered a chance to switch to another door, declines to do so (i.e., he stays with his original choice). Similarly, we say that $C$ is using the "switch" strategy if he picks a door, and, if offered a chance to switch to another door, takes the offer. Now suppose that $C$ decides in advance to play the "stay" strategy. Her only action in this case is to pick a door (and decline an invitation to switch, if one is offered). What is the probability that she wins a car? The same question can be asked about the "switch" strategy. (Idem, p. 137)

Grinstead and Snell remark that the first formulation of the problem "asks for the conditional probability that $\mathrm{C}$ wins if she switches doors, given that she has chosen door 1 and that Monty Hall has chosen door 3" whereas the second formulation is about the comparative probabilities of two kinds of strategies for C, the "switch" strategy and the "stay" strategy. They point out that using the "stay" strategy, the contestant $C$ will win the car with probability $1 / 3$, since $1 / 3$ of the time the door he picks will have the car behind it. But if $C$ plays the "switch" strategy, then he will win whenever the door he originally picked does not have the car behind it, which happens $2 / 3$ of the time. (Idem, p. 13).

Grinstead and Snell give a solution to the first problem using conditional probabilities. van Benthem (2003) gives a solution to the same problem in terms of product updates and probabilites. 
In Sandu (2015b) a modelling of the Monty Hall problem is given using a two player win-lose extensive game of imperfect information. The idea comes from Mann (2010). The emphasis in that paper is on the comparison between different solutions to the Monty Hall problem: on one side the solutions to the first problem above in terms of conditional probabilities and product updates in dynamic logic, and the game-theoretical solution to the second problem, on the other.

The emphasis of the present paper is on the game-theoretical solution to the second problem. We consider several variants of the second problem and model them in IF logic endowed with a probabilistic semantics. The modelization is essentially the same one as in Sandu (2015b) but the overall purpose of the paper is to offer a justification for probabilistic IF logic.

\section{Monty Hall: A game-theoretical modelization}

\subsection{Monty Hall as an extensive game of imperfect infor- mation}

The second formulation of the Monty Hall problem mentioned above refers to strategies. This makes it natural to bring in game-theoretical conceptualizations. We think of Monty Hall and the Contestant as two players, the latter, call her $\mathrm{C}$, trying to identify the door with the prize, whereas her opponent, call him MH, tries to deceive her. The tree which constitutes the extensive form of the game has maximal histories (plays of the game) of the form $(x, y, z, t)$ where:

- $x$ represent the door where MH hides the prize

- $y$ is the door chosen by $C$

- $z$ is the door opened by MH

- $t$ is the second choice of $C$

The rules of the game dictate that

- $z$ must be distinct from $x$ and $y$, and

- $t$ must be distinct from $z$.

Thus the sequence $(1,1,2,1)$ represents the possible play:

MH hides the prize behind door 1; $C$ makes a guess: door 1; $\mathrm{MH}$ opens the empty door 2 ; $C$ guesses again: door 1 .

There are 24 plays (maximal histories). The specification of the player function $P$ which associates with each history other than maximal ones the player whose turn is to move should be quite obvious.

Some of the histories are indistinguishable (informationally equivalent). This holds only for those histories where player $\mathrm{C}$ is to move: 

equivalent for player $C$.

(C1) expresses the fact that $\mathrm{C}$ does not know the door where the prize is hidden when making her first guess. And (C2) epresses the fact that she does not know the door where the prize is hidden, when she makes her second guess.

Each play is winning either for $\mathrm{MH}$ or for $\mathrm{C}$. $\mathrm{C}$ wins (and $\mathrm{MH}$ looses) every play $(x, y, z, t)$ in which she identifies the door which hides the prize, i.e. $t=x$. $\mathrm{MH}$ wins (and $\mathrm{C}$ looses) the remaining ones.

This ends up the description of the game. The result is a 2 player, win-lose finite extensive game of imperfect information.

\subsection{Strategies}

A strategy for player $C$ is any function $F$ which gives her a choice for any history where she is to move. Thus $F$ will give her, for every choice of $x$ by $\mathrm{MH}$, a value for $y$, and for every sequence $(x, y, z)$, a value for $t$. Imperfect information will impose the following restriction (uniformity) on any strategy $F$ :

- If the histories $h$ and $h^{\prime}$ are equivalent, then $F(h)=F\left(h^{\prime}\right)$.

We prefer to decompose any $F$ into two "local" strategies, that is, two functions $f$ and $f^{\prime}$ such that $f$ yields a value for $y$ and $f^{\prime}$ yields a value for $t$. Given (C1), $f$ will be have to be a constant function, that is, a door $y$. Given (C2), $f^{\prime}$ will take only $y$ and and $z$ as arguments.

Thus player C's set $S_{C}$ of strategies will consist of pairs $\left(i, h_{i}\right)$, where $i$ stands for a door and $h_{i}$ for a function of two arguments $(y, z)$. A strategy $\left(i, h_{i}\right)$ is winning if $\mathrm{C}$ wins every play where she follows it. We focus on two kinds of strategies for player $C$.

- The first kind, denoted by $S_{C}^{S t a y}$, groups together all the "stay" strategies, where $\mathrm{C}$ choose a door, and then stick to her initial guess no matter what MH does:

$$
S_{C}^{\text {Stay }}=\left\{\left(i, h_{i}\right): i=1,2,3\right\}
$$

where

$$
h_{i}(y, z)=i
$$

Each strategy $\left(i, h_{i}\right)$ is followed in the play

$$
\left(x, i, z, h_{i}(i, z)\right)
$$

for any $x$ and $z$. There are three "stay" strategies:

$$
\begin{array}{ll}
h_{1}(1,2)=1 & h_{1}(1,3)=1 \\
h_{2}(2,3)=2 & h_{2}(2,1)=2 \\
h_{3}(3,2)=3 & h_{3}(3,1)=3
\end{array}
$$

It should be obvious that each "stay" strategy is winning all the plays where $C$ 's initial guess is correct (i.e., $i=x$ ) and losing the remaining ones. 
- The second kind, denoted by $S_{C}^{\text {Switch }}$ groups together all the "switch" strategies, where $\mathrm{C}$ choose a door, and then, after MH opens a door, switch doors:

$$
S_{C}^{\text {Switch }}=\left\{\left(1, f_{1}\right),\left(2, f_{2}\right),\left(3, f_{3}\right)\right\}
$$

where

$$
\begin{array}{ll}
f_{1}(1,2)=3 & f_{1}(1,3)=2 \\
f_{2}(2,3)=1 & f_{2}(2,1)=3 \\
f_{3}(3,2)=1 & f_{3}(3,1)=2
\end{array}
$$

Given that there are only three doors, each of the three strategies wins all the plays in which the initial choice is incorrect, $i \neq x$, and loses the remaining ones. For illustration, suppose $\mathrm{C}$ follows the strategy $\left(2, f_{2}\right)$. Suppose MH chooses (hides the prize behind) door 1. C's first choice (guess) is door 2. By the rules of the game, MH's next choice can be only door 3 . C's final choice is $f_{2}(2,3)=1$. C wins.

MH's strategies consist also of pairs $(j, g)$ : the first corresponds to a value for $x$; the function $g$ associates to each argument $(x, y)$ a value for $z$.

The only strategy available to $\mathrm{MH}$ (given the rules of the game) is: "hide the prize behind a door, and after $C$ chooses a door, open any other door". Here are all the strategy pairs she can follow:

\begin{tabular}{llll}
$\left(1, g_{1}\right):$ & $g_{1}(1,1)=2$ & $g_{1}(1,2)=3$ & $g_{1}(1,3)=2$ \\
\hline$\left(1, g_{1}^{\prime}\right):$ & $g_{1}^{\prime}(1,1)=3$ & $g_{1}^{\prime}(1,2)=3$ & $g_{1}^{\prime}(1,3)=2$ \\
\hline$\left(2, g_{2}\right):$ & $g_{2}(2,1)=3$ & $g_{2}(2,2)=1$ & $g_{2}(2,3)=1$ \\
\hline$\left(2, g_{2}^{\prime}\right):$ & $g_{2}^{\prime}(2,1)=3$ & $g_{2}^{\prime}(2,2)=3$ & $g_{2}^{\prime}(2,3)=1$ \\
\hline$\left(3, g_{3}\right):$ & $g_{3}(3,1)=2$ & $g_{3}(3,2)=1$ & $g_{3}(3,3)=1$ \\
\hline$\left(3, g_{3}^{\prime}\right):$ & $g_{3}^{\prime}(3,1)=2$ & $g_{3}^{\prime}(3,2)=1$ & $g_{3}^{\prime}(3,3)=2$ \\
\hline
\end{tabular}

Each of the strategy pair $\left(j, g_{j}\right)$ is followed in every play of the form

$$
\left(j, y, g_{j}(j, y), t\right)
$$

for any $y$ and $t$. It is winning whenever $j \neq t$ and losing otherwise. None of these strategies is winning all the plays in which it is followed.

Let us get more dynamics into the game. Let $S_{M H}$ denote the set of strategies of $\mathrm{MH}$ and $S_{C}$ the set of strategies of player C. Whenever $\mathrm{MH}$ follows one of his strategies in $S_{M H}$, and $C$ follows one of her strategies in $S_{C}$, a play of the extensive game is generated which is a win for either one of the players. For instance, when $\mathrm{MH}$ follows $\left(3, g_{3}\right)$ and $\mathrm{C}$ follows $\left(1, h_{1}\right)$, the result is the play $(3,1,2,1)$ which is a win for $\mathrm{MH}$.

The following table registers the payoffs of the players for all the strategy pairs the players might play: 


\begin{tabular}{c|c|c|c|c|c|c|} 
& $\left(1, g_{1}\right)$ & $\left(1, g_{1}^{\prime}\right)$ & $\left(2, g_{2}\right)$ & $\left(2, g_{2}^{\prime}\right)$ & $\left(3, g_{3}\right)$ & $\left(3, g_{3}^{\prime}\right)$ \\
\hline$\left(1, h_{1}\right)$ & $(1,0)$ & $(1,0)$ & $(0,1)$ & $(0,1)$ & $(0,1)$ & $(0,1)$ \\
\hline$\left(2, h_{2}\right)$ & $(0,1)$ & $(0,1)$ & $(1,0)$ & $(1,0)$ & $(0,1)$ & $(0,1)$ \\
\hline$\left(3, h_{3}\right)$ & $(0,1)$ & $(0,1)$ & $(0,1)$ & $(0,1)$ & $(1,0)$ & $(1,0)$ \\
\hline$\left(1, f_{1}\right)$ & $(0,1)$ & $(0,1)$ & $(1,0)$ & $(1,0)$ & $(1,0)$ & $(1,0)$ \\
\hline$\left(2, f_{2}\right)$ & $(1,0)$ & $(1,0)$ & $(0,1)$ & $(0,1)$ & $(1,0)$ & $(1,0)$ \\
\hline$\left(3, f_{3}\right)$ & $(1,0)$ & $(1,0)$ & $(1,0)$ & $(1,0)$ & $(0,1)$ & $(0,1)$ \\
\hline
\end{tabular}

We shall call the game described by the matrix the Monty Hall game. It is a finite, two players, win-lose strategic game

$$
\Gamma_{M H}=\left(S_{C}, S_{M H}, u_{C}, u_{M H}\right)
$$

where $u_{M H}$ and $u_{C}$ are the payoffs of the two players as shown in the matrix.

We can now try to "solve" the game. A natural solution concept to be applied is that of equilibrium. But there is none in the Monty Hall game and we must conclude that the game is indeterminate.

\section{Variants of the Monty Hall game}

We could consider a variant of the Monty Hall game in which the use of the "switch" strategy is built into the winning conditions of the game. The extensive game will be exactly as above, except that the plays $(x, y, z, t)$ won by $\mathrm{C}$ are exactly those for which $x=t$ and $t \neq y$. That is, compared to the previous game, $\mathrm{C}$ wins less plays and $\mathrm{MH}$ wins more in the present variant of the Monty Hall game. The sets $S_{C}, S_{M H}$ of strategies of the two players are exactly the same as in the previous game. However, it is not difficult to see that the "switch" strategies win the same plays as before, whereas the "stay" strategies never win:

\begin{tabular}{c|c|c|c|c|c|c|} 
& $\left(1, g_{1}\right)$ & $\left(1, g_{1}^{\prime}\right)$ & $\left(2, g_{2}\right)$ & $\left(2, g_{2}^{\prime}\right)$ & $\left(3, g_{3}\right)$ & $\left(3, g_{3}^{\prime}\right)$ \\
\hline$\left(1, h_{1}\right)$ & $(0,1)$ & $(0,1)$ & $(0,1)$ & $(0,1)$ & $(0,1)$ & $(0,1)$ \\
\hline$\left(2, h_{2}\right)$ & $(0,1)$ & $(0,1)$ & $(0,1)$ & $(0,1)$ & $(0,1)$ & $(0,1)$ \\
\hline$\left(3, h_{3}\right)$ & $(0,1)$ & $(0,1)$ & $(0,1)$ & $(0,1)$ & $(0,1)$ & $(0,1)$ \\
\hline$\left(1, f_{1}\right)$ & $(0,1)$ & $(0,1)$ & $(1,0)$ & $(1,0)$ & $(1,0)$ & $(1,0)$ \\
\hline$\left(2, f_{2}\right)$ & $(1,0)$ & $(1,0)$ & $(0,1)$ & $(0,1)$ & $(1,0)$ & $(1,0)$ \\
\hline$\left(3, f_{3}\right)$ & $(1,0)$ & $(1,0)$ & $(1,0)$ & $(1,0)$ & $(0,1)$ & $(0,1)$ \\
\hline
\end{tabular}

Like the previous game, this game, let us call it the first variant of the $\mathrm{MH}$ game, is also indeterminate.

Finally, we could consider a second variant of the Monty Hall game in which the use of the "stay" strategy is built into the winning conditions of the game. The extensive game will be exactly as in the two earlier games, except that the plays $(x, y, z, t)$ won by $\mathrm{C}$ are such that $x=t$ and $t=y$. The strategies of the two players are exactly the same as in the previous games. However, in 
this case the "switch" strategies never win whereas the "stay" strategies win the same plays as in the Monty Hall game:

\begin{tabular}{c|c|c|c|c|c|c|} 
& $\left(1, g_{1}\right)$ & $\left(1, g_{1}^{\prime}\right)$ & $\left(2, g_{2}\right)$ & $\left(2, g_{2}^{\prime}\right)$ & $\left(3, g_{3}\right)$ & $\left(3, g_{3}^{\prime}\right)$ \\
\hline$\left(1, h_{1}\right)$ & $(1,0)$ & $(1,0)$ & $(0,1)$ & $(0,1)$ & $(0,1)$ & $(0,1)$ \\
\hline$\left(2, h_{2}\right)$ & $(0,1)$ & $(0,1)$ & $(1,0)$ & $(1,0)$ & $(0,1)$ & $(0,1)$ \\
\hline$\left(3, h_{3}\right)$ & $(0,1)$ & $(0,1)$ & $(0,1)$ & $(0,1)$ & $(1,0)$ & $(1,0)$ \\
\hline$\left(1, f_{1}\right)$ & $(0,1)$ & $(0,1)$ & $(0,1)$ & $(0,1)$ & $(0,1)$ & $(0,1)$ \\
\hline$\left(2, f_{2}\right)$ & $(0,1)$ & $(0,1)$ & $(0,1)$ & $(0,1)$ & $(0,1)$ & $(0,1)$ \\
\hline$\left(3, f_{3}\right)$ & $(0,1)$ & $(0,1)$ & $(0,1)$ & $(0,1)$ & $(0,1)$ & $(0,1)$ \\
\hline
\end{tabular}

This game is indeterminate too.

\section{Logics with imperfect information: Independence- friendly logic (IF logic)}

Hintikka and Sandu (1989) introduce Independence-Friendly logic (IF logic) in order to express more patterns of dependencies (and independencies) of quantifiers than those allowed by ordinary first-order logic. The syntax of IF logic contains quantifers of the form $(\exists x / W)$, and $(\forall x / W)$ where $W$ is a finite set of variables. The intended interpretation of e.g. $(\exists x / W)$ is that the choice of a value for $x$ is independent of the choices of the values for the variables in $W$. When $W=\varnothing$ we recover the standard quantifiers. Alternatively, in terms of the idea of dependence: the choice of a value for $x$ depends on all the values of the variables in whose scope $(\exists x / W)$ is, except for those in $W$.

IF logic is useful for modeling certain puzzles or phenomena of imperfect information. Here is a well known example (Matching Pennies).

Two players, Abelard (the universal player) and Eloise (the existential player) play the following game: Abelard hides one euro in his left or right hand without Eloise seeing it. Eloise has to guess it. If she guesses correctly, she wins; otherwise Abelard wins.

We can model this game in terms of the IF sentence $\varphi_{M P}: \forall x(\exists y /\{x\}) x=y$ and the model set $\mathbb{M}=\{L, R\}$. They determine a semantical game $G\left(\mathbb{M}, \varphi_{M P}\right)$ which is played like this: Abelard chooses $a \in\{L, R\}$ to be the value of the variable $x$. Eloise chooses $b \in\{L, R\}$ to be the value of $y$ without "seeing" the choice of Abelard. Eloise wins the play $(a, b)$ if the assignment $x=a, y=b$ satisfies the quantifier free formula $x=y$, that is, if $a$ is the same as $b$. Otherwise Abelard wins the play.

What is important is not to win a particular play but to have a winning strategy for the whole game. A strategy for a player is a sequence of functions, one for each of his or her move defined on all the possible "known" or "seen" earlier moves. In our semantical game, a strategy for Abelard reduces to an individual of the universe and so does a strategy for Eloise. The set $S_{\forall}$ of strategies for Abelard is thus the same as the set $S_{\exists}$ of strategies for Eloise which is $\mathbb{M}$. Truth and falsity of $\varphi_{M P}$ in $\mathbb{M}$ are defined as expected: 
- $\mathbb{M} \models^{+} \varphi_{M P}$ iff there is a winning strategy for Eloise in $G\left(\mathbb{M}, \varphi_{M P}\right)$ iff there is $b \in\{L, R\}$ such that for all $a \in\{L, R\}: a=b$

- $\mathbb{M} \models^{-} \varphi_{M P}$ iff there is a winning strategy for Abelard in $G\left(\mathbb{M}, s, \varphi_{M P}\right)$ iff there is $a \in\{L, R\}$ such that for all $b \in\{L, R\}: a \neq b$

As we see, we have indeterminacy: $\mathbb{M} \not \not^{+} \varphi_{M P}$ and $\mathbb{M} \not \not^{-} \varphi$.

Notice that were the informal game to have been one of perfect information, that is, were Eloise to see in which hand Abelard hides the coin, we would have modelled it by the semantical game $G(\mathbb{M}, \varphi)$ where $\varphi$ is the ordinary FOL sentence $\forall x \exists y x=y$. In that case $S_{\forall}$ would be, as before, identical to $\mathbb{M}$ but $S_{\exists}$ would consist of four functions (i.e. $(a, a),(b, b),(a, b),(b, a))$. Given that Eloise has more strategies than in the earlier game, $G(\mathbb{M}, \varphi)$ is determined with $(a, b)$ a winning strategy for Eloise. In other words, $\mathbb{M} \models^{+} \varphi$.

If negation occurs in positions other than in front of atomic formulas, then the rules of the semantical game would be changed. In such cases, each occurrence of the negation sign $\neg$ would prompt a swap of the roles of the two players: The moves made "normally" by Eloise would be now made by Abelard, and vice versa. Also the rules for winning and losing would be reversed. One can easily show that the following properties hold for every IF sentence $\varphi$ and model $\mathbb{M}$ :

- $\mathbb{M} \models^{+} \neg \varphi$ if and only if $\mathbb{M} \models^{-} \varphi$

- $\mathbb{M} \models^{-} \neg \varphi$ if and only if $\left.\mathbb{M}\right|^{+} \varphi$.

There is a counterpart of the game-theoretical semantics sketched above which underlies better strategic, game-theoretical phenomena. It is the Skolem semantics. Let us sketch it briefly by way of an example.

With every IF formula $\varphi$ in negation normal form in a given vocabulary $L$ we associate its Skolem form $S k(\varphi)$, which is a first-order formula in an extended vocabulary. Essentially each existentially quantified subformula $(\exists x / W) \psi$ introduces a new Skolem term $f\left(y_{1}, \ldots, y_{n}\right)$ where $y_{1}, \ldots, y_{n}$ are all the free variables of $(\exists x / W) \psi$ minus the variables in $W$. The new function symbol $f$ is intended to codify the "local" strategy of Eloise and the value of the term $f\left(y_{1}, \ldots, y_{n}\right)$ is the individual which is the value of the variable $x$ when $y_{1}, \ldots, y_{n}$ have received appropriate values earlier in the game. Again we prefer to illustrate with an example. Let $\varphi$ be the IF sentence

$$
\exists x \forall y(\exists z /\{y\}) \forall w R(x, y, z, w) .
$$

Its Skolem form $S k(\varphi)$ is

$$
\forall y \forall w R(c, y, f(x), w)
$$

where $c$ is a new 0-place function symbol (constant) and $f$ is a new unary function symbol. The truth of the sentence $\varphi$ on a given model $\mathbb{M}$ in the Skolem semantics is defined as 
- $\mathbb{M} \vDash_{S k}^{+} \varphi$ if and only if there exist an individual $c$ and a function $g$ in the universe of $\mathbb{M}$ to be the interpretations of the new symbols in $S k(\varphi)$ such that

$$
\mathbb{M}, c, g \vDash S k(\varphi) .
$$

The functions $a$ and $g$ are called Skolem functions. We should realize at this point that the Skolem functions are the counterpart of the strategies of Eloise in the semantical game $G(\mathbb{M}, \varphi)$.

Note also that, for an arbitrary IF sentence $\varphi$ in negation normal form, its Skolem form $S k(\varphi)$ and any arbitrary model $\mathbb{M}$ determine the set $S_{\exists}$ of strategies of Eloise in the semantical game $G(\mathbb{M}, \varphi)$ : it is the set of all sequences of Skolem functions which may be the possible interpretations in $\mathbb{M}$ of the new function symbols of $S k(\varphi)$.

The strategies of Abelard in $G(\mathbb{M}, \varphi)$ have also a natural counterpart as Kreisel counterexamples. Again, the details have been given elsewhere (Mann, Sandu and Sevenster, 2011.)

With every IF formula $\varphi$ in negation normal form in a given vocabulary $L$ we associate its Kreisel form $\operatorname{Kr}(\varphi)$, which is also a first-order formula in an extended vocabulary. This procedure is the dual of the procedure of Skolemization. Essentially each universally quantified subformula $(\forall x / W) \psi$ introduces a new Kreisel term $h\left(y_{1}, \ldots, y_{m}\right)$ where $y_{1}, \ldots, y_{m}$ are all the free variables of $(\forall x / W) \psi$ minus the variables in $W$. In our example the Kreisel form of $\varphi$ is

$$
\forall x \forall z \neg R\left(x, h_{1}(x), z, h_{2}(x, f(x), z)\right)
$$

The falsity of $\varphi$ on a given model $\mathbb{M}$ in the Skolem semantics is defined as:

- $\mathbb{M} \vDash_{S k}^{-} \varphi$ if and only if there exist function $g_{1}$ and $g_{2}$ in $\mathbb{M}$ to be the interpretations of the new function symbols in $\operatorname{Kr}(\varphi)$ such that

$$
\mathbb{M}, g_{1}, g_{2} \vDash K r(\varphi)
$$

We call $g_{1}$ and $g_{2}$ Kreisel counterexamples.

Mann, Sandu and Sevenster (2011) gives a detailed introduction to game-theoretical semantics, Skolem semantics and the compositional semantics and shows their equivalence.

\section{Some basic results on IF prefixes}

In this section we describe some of the results in Sevenster (2014) which help us to evaluate the expressive power of IF quantifier prefixes.

An IF prefix is a series $\left(Q_{1} x_{1} / U_{1}\right) \ldots\left(Q_{n} x_{n} / U_{n}\right)$ of quantifiers, where $Q_{i}$ is either $\exists$ or $\forall, u_{i}$ a variable and $U_{i}$ is a finite set of variables disjoint from $u_{i}$. We require that all quantified variables $x_{i}$ in an IF prefix be distinct.

A prefix $\left(Q_{1} x_{1} / U_{1}\right) \ldots\left(Q_{n} x_{n} / U_{n}\right)$ is sentential if $U_{i} \subseteq\left\{u_{1}, \ldots, u_{i-1}\right\}$ for every $1 \leq i \leq n$. An IF formula $\Pi \varphi$ is a sentence if the prefix $\Pi$ is sentential and all variables appearing in $\varphi$ are contained in the set of variables quantified in $\Pi$. 
Let $\varphi$ and $\psi$ be two IF sentences. We say that $\varphi$ and $\psi$ are (truth) equivalent, if for every suitable structure $\mathbb{M}$ we have,

$$
\mathbb{M} \models^{+} \varphi \quad \text { iff }\left.\quad \mathbb{M}\right|^{+} \psi .
$$

Let $\Pi$ and $\Phi$ be IF prefixes. Then, $\Pi$ and $\Phi$ are equivalent if $\Pi \varphi$ and $\Phi \varphi$ are equivalent for every quantifier-free formula $\varphi$.

The IF formalism introduces a difference between syntactical scope and semantical scope (dependence). Let $Q x / X$ and $Q y / Y$ be two quantifiers in an IF formula such that $Q x / X$ is in the (syntactical) scope of $Q y / Y$. We say that $Q x / X$ depends on $Q y / Y$ if $y \notin X$. Notice that if $X=\varnothing$, then $Q x$ depends on $Q y / Y$. Two patterns of dependence turn out to be important for expressive power:

- An IF prefix $\Pi$ is a Henkin prefix if it contains four quantifiers

$$
(\forall x / X),(\forall y / Y),(\exists u / U),(\exists v / V)
$$

such that $(\exists u / U)$ depends on $x$ and not on $y$; and $(\exists v / V)$ depends on $y$ and not on $x$ and $u$. Notice that the first condition requires $(\exists u / U)$ to be in the syntactical scope of $(\forall x / X)$ and the second condition requires $(\exists v / V)$ to be in the syntactical scope of $(\forall y / Y)$. For example

$$
\forall x \forall y(\exists u /\{y\})(\exists v /\{x, u\})
$$

is a Henkin prefix and so is

$$
\forall x \exists u \forall y(\exists v /\{x, u\}) .
$$

- An IF prefix $\Pi$ is a signaling prefix if it contains three quantifiers

$$
(\forall u / U),(\exists v / V),(\exists w / W)
$$

syntactically ordered as above such that $(\exists v / V)$ depends on $u$ and $(\exists w / W)$ depends on $v$ but not on $u$. For example

$$
\forall u \exists v(\exists w /\{u\})
$$

is a signaling prefix.

Sevenster (2014) considers three operations on prefixes that preserve truth equivalence.

The first operation is quantifier swapping. Every two prefixes

$$
\Pi(Q u / U)(Q v / V \cup u) \Pi^{\prime}
$$


and

$$
\Pi(Q v / V-u)(Q u / U \cup v) \Pi^{\prime}
$$

of IF quantifiers are equivalent.

The second operation is emptying the slash set from an universal quantifier. Every two prefixes

$$
(\forall v / V) \Pi \text { and } \forall v \Pi
$$

of IF quantifiers are equivalent.

The third operation consists of emptying an existentially quantified slash set. Every two sentential prefixes

$$
\Pi(Q v / V) \Pi^{\prime}
$$

and

$$
\Pi Q v \Pi^{\prime}
$$

of IF quantifiers are equivalent whenever $V$ contains variables that are quantified only by existential quantifiers in $\Pi$.

A prefix $\Pi$ of IF quantifiers is called primary if it is neither Henkin nor signaling.

Lemma (Sevenster 2014) Let $\Pi$ be a IF prefix and let $\Pi_{0}$ be the IF prefix that is the result of swapping two quantifiers in (as in the first operation above ); or emptying the slash set of a universal quantifier in $\Pi$ (as in the second operation above); or emptying the slash set of a quantifier in $\Pi$ that contains only existentially quantified variables (as in the third operation above). Then, $\Pi_{0}$ is primary, whenever $\Pi$ is.

Lemma (Sevenster 2014) Every primary, sentential IF prefix $\Pi(\exists v / V) \Pi^{\prime}$ in which $\Pi$ is an FOL prefix, is equivalent to a primary, sentential IF prefix $\Sigma \Pi^{\prime}$ in which $\Sigma$ is an FOL prefix.

Using these two Lemmas, Sevenster (2014) shows:

Theorem Every prefix which is neither signaling nor Henkin is (truth) equivalent to an ordinary FOL prefix.

\section{Defining the Monty Hall games in IF logic}

We shall consider three IF sentences,

$$
\begin{gathered}
\varphi_{M H}=: \forall x(\exists y /\{x\}) \forall z(\exists t /\{x\})[x \neq z \wedge y \neq z \rightarrow t=x] \\
\varphi_{M H 1}=: \forall x(\exists y /\{x\}) \forall z(\exists t /\{x\})[x \neq z \wedge y \neq z \rightarrow(t \neq y \wedge x=t)] \\
\varphi_{M H 2}=: \forall x(\exists y /\{x\}) \forall z(\exists t /\{x\})[x \neq z \wedge y \neq z \rightarrow(t=y \wedge x=t)] .
\end{gathered}
$$

We can think of the Contestant, $\mathrm{C}$, as the existential quantifier and of $\mathrm{MH}$ as the universal quantifier. Let $\mathbb{M}$ be a model set which consists of three doors. Any of the three sentences $\varphi \in\left\{\varphi_{M H}, \varphi_{M H 1}, \varphi_{M H 2}\right\}$ and the model $\mathbb{M}$ determine a semantical game $G(\mathbb{M}, \varphi)$, as we have seen. In such a game, the two players 
choose individuals from the set $\mathbb{M}$ to be the values of the variables $x, y, z$ and $t$. A play of any of these games is thus a sequence of four individuals in $\mathbb{M}$. For instance, the sequence $(1,2,3,1)$ is intended to represent the play:

MH hides the prize behind door $1 ; C$ makes a guess: door 2; $\mathrm{MH}$ opens door 3 ; $C$ guesses again: door 1 .

The games $G\left(\mathbb{M}, \varphi_{M H}\right), G\left(\mathbb{M}, \varphi_{M H 1}\right)$ and $G\left(\mathbb{M}, \varphi_{M H 2}\right)$ have the same set of histories. They differ only in the winning conditions (payoffs) of the maximal histories (plays) that are determined in each case by the relevant quantifier free subformula. For instance, the play $(1,2,3,1)$ determines the assignment $x=$ $1, y=2, z=3$ and $t=1$ which satisfies the quantifier free subformula of $\varphi_{M H}$. We conclude that the play is a win for $\mathrm{C}$. As the assignment determined by any play of any of the games either satisfies the relevant quantifier-free subformula or not, it is obvious that $G\left(\mathbb{M}, \varphi_{M H}\right), G\left(\mathbb{M}, \varphi_{M H 1}\right)$ and $G\left(\mathbb{M}, \varphi_{M H 3}\right)$ are 2 player, win-lose extensive game of imperfect information. We shall take them to "define" in IF logic the three games discussed in an earlier section.

Strictly speaking, the semantical games do not correspond exactly to the earlier Monty Hall game and its two variants. But they are "equivalent" to them. To see this, let us consider the Monty Hall game and the semantical game $G\left(\mathbb{M}, \varphi_{M H}\right)$ that we take to define it. The definitory rules of the Monty Hall game are built into the winning conditions of the semantical game and the rationality assumptions behind it. Take the rule that $z$ must be distinct from $x$ and $y$. In the semantical game, if, for instance, $\mathrm{MH}$ chooses to open either the door $x$ where he hid the price or the door $y$ chosen by $\mathrm{C}$, he will lose right away. Therefore if $\mathrm{MH}$ is a rational player (and we assume he is), he will choose to open a door $z$ such that $z \neq x$ and $z \neq y$. Similarly for the other definitory rule which prescribes that $t$ must be distinct than $z$. In the semantical game nothing in the rules of the game prevents $\mathrm{C}$ from choosing $t$ to be the same as $z$. But we already pointed out that it is in MH's interest to choose $z$ distinct from $x$. And as $\mathrm{C}$ is rational herself, and she knows that $\mathrm{MH}$ is rational, it is not in her interest to choose $t$ to be the same as $z$. All these considerations have a precise game-theoretical counterpart: such "irrational" strategies turn out to be weakly dominated by other strategies of the same player (cf. below.)

The information sets of the two players will be determined by the same principles (C1) and (C2) that we discussed earlier.

The strategies of the two players in a semantical game are determined by the Skolem and Kreisel form of the relevant IF sentence and the underlying model set, as we pointed out earlier. For instance the Skolem form of $\varphi_{M H}$ is

$$
\forall x \forall z[x \neq z \wedge c \neq z \rightarrow f(y, z)=x]
$$

and its Kreisel form is

$$
\forall y \forall t[d \neq g(x, y) \wedge y \neq g(x, y) \wedge t \neq d]
$$

Therefore we shall take C's strategies to consist of all pairs $\left(i, f_{i}\right)$, such that $i \in M$ and $f_{i}: M^{2} \rightarrow M$. And the strategies of Monty Hall will be also pairs 
$\left(j, g_{j}\right)$ such that $j \in M$ and $g_{j}: M^{2} \rightarrow M$. These are also the strategies of $\mathrm{C}$ and respectively $\mathrm{MH}$ in the two other semantical games.

\subsection{The semantical indeterminacy of the Monty Hall sen- tences}

We will show that $\varphi_{M H}, \varphi_{M H 1}$ and $\varphi_{M H 2}$ are semantically indeterminate on the model set $\mathbb{M}$ with three elements. There are at least three ways to do this.

One way would be to reason as follows. We have established that $G\left(\mathbb{M}, \varphi_{M H}\right), G\left(\mathbb{M}, \varphi_{M H 1}\right)$ and $G\left(\mathbb{M}, \varphi_{M H 2}\right)$ define the Monty Hall game and its variants. Given that the latter are indeterminate in the game theoretical sense, that is, there is no equilibrium in the games, then it also follows that that the former are indeterminate in the semantical sense. This argument would require that we further establish an equivalence between the lack of an equilibrium in the latter games and and the lack of a winning strategy for either player in the semantical game. Such an argument for the general case may be found in Barbero and Sandu (2014).

A second way would be to use the definitions of truth and falsity in Skolem semantics to show that $\varphi_{M H}, \varphi_{M H 1}$ and $\varphi_{M H 2}$ are semantically indeterminate in $\mathbb{M}$. The definitions tells us that:

- $\mathbb{M} \vDash_{S k}^{+} \varphi_{M H}$ iff there exist $i \in M$ and a function $f_{i}$ in $\mathbb{M}$ such that

$$
\mathbb{M}, i, f_{i} \vDash \forall x \forall z[x \neq z \wedge c \neq z \rightarrow f(y, z)=x]
$$

and

- $\mathbb{M} \vDash_{S k}^{-} \varphi_{M H}$ iff there exist $j \in M$ and a function $g_{j}$ in $\mathbb{M}$ such that

$$
\mathbb{M}, j, g_{j} \vDash \forall y \forall t[d \neq g(x, y) \wedge y \neq g(x, y) \wedge t \neq d]
$$

We could then show that none of the conditions on the right side of the equivalences holds.

We prefer to apply a simpler, third alternative procedure based on Sevenster's result described earlier. We first notice that each of the three prefixes is neither branching nor signaling. By Sevenster's theorem, each of the three sentences is truth equivalent with an ordinary FOL sentence. In the case of $\varphi_{M H 1}$

$$
\forall x(\exists y /\{x\}) \forall z(\exists t /\{x\})[x \neq z \wedge y \neq z \rightarrow(t \neq y \wedge x=t)]
$$

we obtain its first-order equivalent by the following steps.

- First we swap the first two quantifiers:

$$
\exists y(\forall x /\{x)\}) \forall z(\exists t /\{x\})[x \neq z \wedge y \neq z \rightarrow(t \neq y \wedge x=t)] .
$$

- We then empty the slash set of the universal quantifier in the resulting sentence:

$$
\exists y \forall x \forall z(\exists t /\{x\})[x \neq z \wedge y \neq z \rightarrow(t \neq y \wedge x=t)] .
$$


- Next we swap the two universal quantifiers:

$$
\exists y \forall z \forall x(\exists t /\{x\})[x \neq z \wedge y \neq z \rightarrow(t \neq y \wedge x=t)]
$$

- We swap the last two quantifiers:

$$
\exists y \forall z \exists t(\forall x /\{t\})[x \neq z \wedge y \neq z \rightarrow(t \neq y \wedge x=t)] .
$$

- Finally we empty the slashed set of the universal quantifier:

$$
\exists y \forall z \exists t \forall x[x \neq z \wedge y \neq z \rightarrow(t \neq y \wedge x=t)] .
$$

Let us denote this sentence by $\varphi_{M H 1}^{+}$. By Sevenster's result, $\varphi_{M H 1}$ and $\varphi_{M H 1}^{+}$ are truth equivalent on all models. In particular, they are truth equivalent on our model set $\mathbb{M}$ with three elements. However it is easy to show that the first-order sentence $\varphi_{M H 1}^{+}$is not true in $\mathbb{M}$ (i.e. it is false in $\mathbb{M}$ ). We conclude that

$$
\mathbb{M} \not \nvdash^{+} \varphi_{M H 1} .
$$

On the other side, from the game-theoretical semantics of IF logic, we know that

$$
\mathbb{M}=^{-} \varphi_{M H 1} \quad \text { iff } \quad \mathbb{M} \models^{+} \neg \varphi_{M H 1} .
$$

But $\neg \varphi_{M H 1}$ is truth equivalent with the IF sentence which is obtained by pushing negation infront of the atomic formulas in such a way that every quantifier and connective is changed into its dual. In our case this sentence, let us denote it by $\varphi_{M H 1}^{*}$, is

$$
\exists x(\forall y /\{x\}) \exists z(\forall t /\{x\})[x \neq z \wedge y \neq z \wedge(x \neq t \vee t=y)] .
$$

Applying Sevenster's transformations, $\varphi_{M H 1}^{*}$ can be shown to be equivalent to the first-order sentence

$$
\exists x \forall y \exists z \forall t[x \neq z \wedge y \neq z \wedge(x \neq t \vee t=y)] .
$$

This sentence is false in $\mathbb{M}$. Thus

$$
\mathbb{M} \not \not^{+} \varphi_{M H 1} \quad \text { and } \quad \mathbb{M} \not \nvdash^{\prime}-\varphi_{M H 1} .
$$

A similar argument also shows that

$$
\mathbb{M} \not \nvdash^{+} \varphi_{M H 2} \quad \text { and } \quad \mathbb{M} \not \nvdash^{-} \varphi_{M H 2}
$$

and

$$
\mathbb{M} \not \nvdash^{+} \varphi_{M H} \quad \text { and } \quad \mathbb{M} \not \nvdash^{-} \varphi_{M H} .
$$

(We are indebted to Barbero for the above argument). We have reached the conclusion that $\varphi_{M H}, \varphi_{M H 1}$ and $\varphi_{M H 2}$ are neither true nor false on $\mathbb{M}$. Of course this semantic indeterminacy mirrors the lack of pure strategy equilibria in the three Monty Hall games discussed earlier. 
The question which interests us now is: Is their a way to distinguish between $\varphi_{M H}, \varphi_{M H 1}$ and $\varphi_{M H 2}$ ? Of course the distinction cannot be spelled out in terms of truth-functional content, that is, by evaluating what the three sentences "assert" or "exclude": for, as the above argument showed, what these sentences assert is false, and what they exclude is also false. Still, there are differences between them. Consider, for instance, $\varphi_{M H 1}$ and $\varphi_{M H 2}$ : the condition $(t \neq$ $y \wedge x=t$ ) makes the "switch" strategy weakly dominant, whereas the condition $(t=y \wedge x=t)$ makes the "stay" strategy to be so. Is there a way to incorporate this difference into the semantical values of the two sentences?

The framework which yields the desired result has been worked out for the first time in Sevenster (2006), developed in Sevenster and Sandu (2010), and Mann, Sandu and Sevenster (2011). The application of this framework to Monty Hall has been given for the first time by Mann (2010), and developed in Sandu (2015).

\section{Mixed strategy equilibria: von Neumann's Min- imax Theorem}

We shall overcome the indetermincy of the three games above by an appeal to a technique which is well known in classical game theory: von Neumann's Minimax Theorem.

Let

$$
\Gamma=\left(S_{\exists}, S_{\forall}, u_{\exists}, u_{\forall}\right)
$$

be a two player, win-lose, finite strategic game, where $S_{p}$ is the set of pure strategies of player $p$. A mixed strategy $\nu$ for player $p$ is a probability distribution over $S_{p}$, that is, a function $\nu: S_{p} \rightarrow[0,1]$ such that $\sum_{\tau \in S_{i}} \nu(\tau)=1 . \nu$ is uniform over $S_{i}^{\prime} \subseteq S_{i}$ if it assigns equal probability to all strategies in $S_{i}^{\prime}$ and zero probability to all the strategies in $S_{i}-S_{i}^{\prime}$. The support of $\nu$ is the set of strategies to which $\nu$ assigns non-zero probability. Obviously we can simulate a pure strategy $\sigma$ with a mixed strategy $\nu$ such that $\nu$ assigns $\sigma$ probability 1 . Given a mixed strategy $\mu$ for player $\exists$ and a mixed strategy $\nu$ for player $\forall$, the expected utility for player $p$ is given by:

$$
U_{p}(\mu, \nu)=\sum_{\sigma \in S_{\exists}} \sum_{\tau \in S_{\forall}} \mu(\sigma) \nu(\tau) u_{p}(\sigma, \tau) .
$$

Let $\mu$ be a mixed strategy for player $\exists$ and $\nu$ a mixed strategy for player $\forall$. The pair $(\mu, \nu)$ is an equilibrium if the following two conditions hold:

1. for every mixed strategy $\mu^{\prime}$, of Eloise, $U_{\exists}(\mu, \nu) \geq U_{\exists}\left(\mu^{\prime}, \nu\right)$

2. for every mixed strategy $\nu^{\prime}$ of Abelard, $U_{\forall}(\mu, \nu) \geq U_{\forall}\left(\mu, \nu^{\prime}\right)$.

The following two results are well known.

Theorem (von Neuman's Minimax Theorem; von Neumann 1928) Every finite, two-person, constant-sum game has an equilibrium in mixed strategies. 
Corollary Let $(\mu, \nu)$ and $\left(\mu^{\prime}, \nu^{\prime}\right)$ be two mixed strategy equlibria in a constant sum game. Then $U_{p}(\mu, \nu)=U_{p}\left(\mu^{\prime}, \nu^{\prime}\right)$

These two results guarantee that we can talk about the value $V(\Gamma)$ of a strategic game $\Gamma$ : it is the expected utility returned to player $\exists$ by any equilibrium in the relevant strategic game.

The next results will help us to identify equilibria. Their proof may be found in Mann, Sandu and Sevenster (2011), chapter 7.

We start with a couple of definitions.

Let $\Gamma=\left(S_{\exists}, S_{\forall}, u_{\exists}, u_{\forall}\right)$ be a strategic game where $S_{p}$ is the set of pure strategies of player $p$. For $\sigma, \sigma^{\prime} \in S_{\exists}$, we say that $\sigma^{\prime}$ weakly dominates $\sigma$ if the following two conditions hold:

(i) For every $\tau \in S_{\forall}: u_{\exists}\left(\sigma^{\prime}, \tau\right) \geq u_{\exists}(\sigma, \tau)$

(ii) For some $\tau \in S_{\forall}: u_{\exists}\left(\sigma^{\prime}, \tau\right)>u_{\exists}(\sigma, \tau)$.

A similar notion is defined for Abelard.

We say that $\sigma^{\prime}$ is payoff equivalent to $\sigma$ if for every $\tau \in S_{\forall}: u_{\exists}\left(\sigma^{\prime}, \tau\right)=$ $u_{\exists}(\sigma, \tau)$.

A similar notion is defined for Abelard.

We list three results from the game-theoretical literature. The first one enables one to identify equilibria in mixed strategies. It is given here in order to illustrate how indeterminate IF sentences can be distinguished by their probabilistic semantics. The other two enable us to eliminate weakly dominated strategies and payoff equivalent ones. They will be applied to the Monty Hall sentence.

For the first result we recall that we can simulate a pure strategy with a mixed strategy which assigns 1 to that pure strategy and 0 to the rest.

Then for $\sigma \in S_{\exists}$ and $\nu$ a mixed strategy for Abelard we have:

$$
U_{p}(\sigma, \nu)=\sum_{\tau \in S_{\forall}} \nu(\tau) u_{p}(\sigma, \tau) .
$$

Similarly, for $\tau \in S_{\forall}$ and $\mu$ a mixed strategy for Eloise, we have

$$
U_{p}(\mu, \tau)=\sum_{\sigma \in S_{\exists}} \mu(\sigma) u_{p}(\sigma, \tau) .
$$

Proposition Let $\mu^{*}$ be a mixed strategy for Eloise and $\nu^{*}$ a mixed strategy for Abelard in a finite, two player strategic game $\Gamma$. The pair $\left(\mu^{*}, \nu^{*}\right)$ is an equilibrium in $\Gamma$ if and only if the following conditions hold:

1. $U_{\exists}\left(\mu^{*}, \nu^{*}\right)=U_{\exists}\left(\sigma, \nu^{*}\right)$ for every $\sigma \in S_{\exists}$ in the support of $\mu^{*}$

2. $U_{\exists}\left(\mu^{*}, \nu^{*}\right)=U_{\forall}\left(\mu^{*}, \tau\right)$ for every $\tau \in S_{\forall}$ in the support of $\nu^{*}$

3. $U_{\exists}\left(\mu^{*}, \nu^{*}\right) \geq U_{\forall}\left(\sigma, \nu^{*}\right)$ for every $\sigma \in S_{\exists}$ outside the support of $\mu^{*}$

4. $U_{\forall}\left(\mu^{*}, \nu^{*}\right) \geq U_{\forall}\left(\mu^{*}, \tau\right)$ for every $\tau \in S_{\forall}$ outside the support of $\nu^{*}$. 
Proof. See Osborne 2003, p. 116.

Here is a well known example in the IF literature (see e.g. Sevenster, 2006), where the above proposition is applied. We compare two strategic IF games, $\Gamma\left(\mathbb{M}, \varphi_{M P}\right)$ and $\Gamma\left(\mathbb{M}, \varphi_{I M P}\right)$ where $\mathbb{M}=\{1,2,3,4\}, \varphi_{M P}$ is the Matching Pennies sentence $\forall x(\exists y /\{x\}) x=y$ and $\varphi_{I M P}$ is the Inverted Matching Pennies sentence $\forall x(\exists y /\{x\}) x \neq y$. The first game is represented on the left and the second on the right:

\begin{tabular}{c|c|c|c|c|} 
& $\tau_{1}$ & $\tau_{2}$ & $\tau_{3}$ & $\tau_{4}$ \\
\hline$\sigma_{1}$ & $(1,0)$ & $(0,1)$ & $(0,1)$ & $(0,1)$ \\
\hline$\sigma_{2}$ & $(0,1)$ & $(1,0)$ & $(0,1)$ & $(0,1)$ \\
\hline$\sigma_{3}$ & $(0,1)$ & $(1,0)$ & $(1,0)$ & $(0,1)$ \\
\hline$\sigma_{4}$ & $(0,1)$ & $(0,1)$ & $(0,1)$ & $(1,0)$ \\
\hline
\end{tabular}

\begin{tabular}{c|c|c|c|c|} 
& $\tau_{1}$ & $\tau_{2}$ & $\tau_{3}$ & $\tau_{4}$ \\
\hline$\sigma_{1}$ & $(0,1)$ & $(1,0)$ & $(1,0)$ & $(1,0)$ \\
\hline$\sigma_{2}$ & $(1,0)$ & $(0,1)$ & $(1,0)$ & $(1,0)$ \\
\hline$\sigma_{3}$ & $(1,0)$ & $(1,0)$ & $(0,1)$ & $(1,0)$ \\
\hline$\sigma_{4}$ & $(1,0)$ & $(1,0)$ & $(1,0)$ & $(0,1)$ \\
\hline
\end{tabular}

We show that the strategy pair $\left(\mu^{*}, \nu^{*}\right)$ where both $\mu^{*}$ and $\nu^{*}$ are uniform strategies with suport $M=\{1,2,3,4\}$. First notice that in the first game $U_{\exists}\left(\mu^{*}, \nu^{*}\right)=1 / 4$ and thus $U_{\forall}\left(\mu^{*}, \nu^{*}\right)=3 / 4$. Notice that clauses (3) and (4) of the above Proposition are trivially satisfied. For clause (1), consider $\sigma_{1}$ and recall the equation

$$
U_{\exists}\left(\sigma_{1}, \nu^{*}\right)=\sum_{\tau \in S_{I I}} \nu^{*}(\tau) u_{I}\left(\sigma_{1}, \tau\right)
$$

to get $U_{\exists}\left(\sigma_{1}, \nu^{*}\right)=1 / 4$. A similar computation establishes clause (2). The argument for the second game is similar.

Proposition Let $\Gamma=\left(S_{\exists}, S_{\forall}, u_{\exists}, u_{\forall}\right)$ be a 2 player, finite, win-lose strategic game. Then $\Gamma$ has a mixed strategy equilibrium $(\mu, \nu)$ such that for each player $p$ none of the pure strategies in the support of his mixed strateggy is weakly dominated in $\Gamma$. (The proof requires the finiteness of the game).

Proposition Let $\Gamma=\left(S_{\exists}, S_{\forall}, u_{\exists}, u_{\forall}\right)$ be a 2 player, finite, win-lose strategic IF game. Then $\Gamma$ has a mixed strategy equilibrium $(\mu, \nu)$ such that for each player there are no pure strategies in the support of his mixed strategy which are payoff equivalent.

We apply these results to the Monty Hall game. The second proposition allows us to reduce the game to the smaller one:

\begin{tabular}{c|c|c|c|} 
& $\left(1, g_{1}\right)$ & $\left(2, g_{2}\right)$ & $\left(3, g_{3}\right)$ \\
\hline$\left(1, h_{1}\right)$ & $(1,0)$ & $(0,1)$ & $(0,1)$ \\
\hline$\left(2, h_{2}\right)$ & $(0,1)$ & $(1,0)$ & $(0,1)$ \\
\hline$\left(3, h_{3}\right)$ & $(0,1)$ & $(0,1)$ & $(1,0)$ \\
\hline$\left(1, f_{1}\right)$ & $(0,1)$ & $(1,0)$ & $(1,0)$ \\
\hline$\left(2, f_{2}\right)$ & $(1,0)$ & $(0,1)$ & $(1,0)$ \\
\hline$\left(3, f_{3}\right)$ & $(1,0)$ & $(1,0)$ & $(0,1)$ \\
\hline
\end{tabular}


We notice that each "stay" strategy is weakly dominated by some "switch" strategy. We apply the first proposition and reduce the game to:

\begin{tabular}{l|c|c|c|} 
& $\left(1, g_{1}\right)$ & $\left(2, g_{2}\right)$ & $\left(3, g_{3}\right)$ \\
\hline$\left(1, f_{1}\right)$ & $(0,1)$ & $(1,0)$ & $(1,0)$ \\
\hline$\left(2, f_{2}\right)$ & $(1,0)$ & $(0,1)$ & $(1,0)$ \\
\hline$\left(3, f_{3}\right)$ & $(1,0)$ & $(1,0)$ & $(0,1)$ \\
\hline
\end{tabular}

Let $\mu$ be the uniform probability distribution $\mu\left(1, f_{i}\right)=1 / 3$ and $\nu$ the uniform probability distribution $\nu\left(j, g_{j}\right)=1 / 3$. It may be shown that this is an equilibrium in the game and that the expected utility of player $C$ for this equilibrium is $2 / 3$.

We consider the first variant of the MH game. By applying the two propositions above we obtain the game described in the last paragraph. Hence it's value is the same, $2 / 3$.

As for the last game, after applying the two propositions we reduce it to

\begin{tabular}{c|c|c|c|} 
& $\left(1, g_{1}\right)$ & $\left(2, g_{2}\right)$ & $\left(3, g_{3}\right)$ \\
\hline$\left(1, h_{1}\right)$ & $(1,0)$ & $(0,1)$ & $(0,1)$ \\
\hline$\left(2, h_{2}\right)$ & $(0,1)$ & $(1,0)$ & $(0,1)$ \\
\hline$\left(3, h_{3}\right)$ & $(0,1)$ & $(0,1)$ & $(1,0)$ \\
\hline
\end{tabular}

Let $\mu$ be the uniform probability distribution $\mu\left(1, h_{i}\right)=1 / 3$ and $\nu$ the uniform probability distribution $\nu\left(j, g_{j}\right)=1 / 3$. It may be shown that this is an equilibrium in the game and that the expected utility of player $C$ for this equilibrium is $1 / 3$.

\section{Some general remarks}

We shall take the probabilistic value of an IF sentence $\varphi$ on a finite model $\mathbb{M}$ to be the value of the 2 player, win-lose, finite strategic game which can be obtained from $G(\mathbb{M}, \varphi)$ along the procedure illustrated in the preceding section. It is the expected utility returned to Eloise by any of the mixed strategy equilibria in this game. The existence of such an equilibrium is guaranteed by von Neumann's Minimax theorem. We denote the probabilistic value by $P(\varphi, \mathbb{M})$. Mann, Sandu and Sevenster (2011) collect the basic results on probabilistic IF logic. They prove, among other things, the following:

P1

$$
P(\varphi \vee \psi, \mathbb{M})=\max (P(\varphi, \mathbb{M}), P(\psi, \mathbb{M})) .
$$

$\mathrm{P} 2$

$$
P(\varphi \wedge \psi, \mathbb{M})=\min (P(\varphi, \mathbb{M}), P(\psi, \mathbb{M})) \text {. }
$$

P3

$$
P(\neg \varphi, \mathbb{M})=1-P(\varphi, \mathbb{M}) \text {. }
$$

P4

$$
P(\exists x \varphi, \mathbb{M})=\max \{P(\varphi, b, \mathbb{M}): b \in M\} \text {. }
$$

P5

$$
P(\forall x \varphi, \mathbb{M})=\min \{P(\varphi, b, \mathbb{M}): b \in M\} \text {. }
$$


In $\mathrm{P} 4$ and $\mathrm{P} 5 \exists x \varphi$ and $\forall x \varphi$ are sentences and $P(\varphi, b, \mathbb{M})$ is the value of strategic game $\Gamma(\mathbb{M}, s, \varphi)$ where $s$ is the assignment which assigns $b$ to $\mathrm{x}$.

Let us give an example which illustrates how we compute the probabilistic value of an IF sentence form the probabilistic values of its subformulas. The example if from Mann, Sandu and Sevenster 2011, p. 168. Consider the IF sentence $\psi$

$$
\exists u \forall w\left(u \neq w \vee \varphi_{M P}\right)
$$

on a finite model-set $\mathbb{M}$ with $n$ elements. Here $\varphi_{M P}$ is the Matching Pennies sentence.

$\mathrm{P} 4$ and $\mathrm{P} 5$ tell us that

$$
N E(\psi, \mathbb{M})=\max _{a} \min _{b}\left\{N E\left(u \neq w \vee \varphi_{M P},(u, a),(w, b), \mathbb{M}\right): a, b \in M\right\} .
$$

By P1, we know that

$$
\begin{aligned}
& N E\left(u \neq w \vee \varphi_{M P},(u, a),(w, b), \mathbb{M}\right) \\
& \max \left(N E(u \neq w,(u, a),(w, b), \mathbb{M}), N E\left(\varphi_{M P},(u, a),(w, b), \mathbb{M}\right)\right) .
\end{aligned}
$$

We know already that $N E\left(\varphi_{M P},(u, a),(w, b), \mathbb{M}\right)$ is $1 / n$. By P1 we get

$$
N E\left(u \neq w \vee \varphi_{M P},(u, a),(w, b), \mathbb{M}\right)=\max (N E(u \neq w,(u, a),(w, b), \mathbb{M}), 1 / n) .
$$

From (E1) and (E2) we know that $N E(u \neq w,(u, a),(w, b), \mathbb{M})$ is 1 if $a \neq b$ and 0 otherwise. Thus for a fixed $a, \min _{b}\left\{N E\left(u \neq w \vee \varphi_{M P},(u, a),(w, b), M\right): b \in M\right\}$ is reached when $w$ is $a$ and this minimum is $1 / n$. We conclude that $\max _{a} \min _{b}$ is $1 / 2$.

On the basis of P1-P3 it is straightforward to show that the following hold for any IF sentence $\varphi$ and finite model $\mathbb{M}$ :

Ax1

$$
P(\varphi, \mathbb{M}) \geq 0
$$

Ax2

$$
P(\varphi, \mathbb{M})+P(\neg \varphi, \mathbb{M})=1
$$

Ax3

$$
P(\varphi, \mathbb{M})+P(\psi, \mathbb{M}) \geq P(\varphi \vee \psi, \mathbb{M})
$$

$\mathrm{Ax} 4$

$$
P(\varphi \wedge \psi, \mathbb{M})=0 \rightarrow P(\varphi, \mathbb{M})+P(\psi, \mathbb{M})=P(\varphi \vee \psi, \mathbb{M}) .
$$

The axioms are known as Kolmogorov axioms of probabilities.

It has been pointed out that there is another principle which may be considered natural in the context of logic:

S. (Substitutivity) If $\varphi$ and $\psi$ are truth equivalent, then $P(\varphi, \mathbb{M})=P(\psi, \mathbb{M})$ for every finite model $\mathbb{M}$.

The IF sentence $\varphi_{M H}$ and the first-order sentence $\exists y \forall z \exists t \forall x[x \neq z \wedge y \neq z \rightarrow$ $x=t]$ which is equivalent with it provide a counter-example to (S). On any model $\mathbb{M}$ with at least three elements, $P\left(\varphi_{M H}, \mathbb{M}\right) \neq 0, P\left(\varphi_{M H}, \mathbb{M}\right) \neq 1$ but

$$
P(\exists y \forall z \exists t \forall x[x \neq z \wedge y \neq z \rightarrow x=t], \mathbb{M})=0 .
$$

For a more detailed comparison between IF probabilities and other kinds of probabilistic semantics we refer to Sandu (forthcoming 2015b). 


\section{Bibliography}

F. Barbero, 'On existential declarations of independence in IF Logic', The Review of Symbolic Logic, 6, 254-280, 2013.

F. Barbero and G. Sandu, 'Signaling in Independence-Friendly Logic', Logic Jnl IGPL, 22, 638-664, 2014, doi: 10.1093/jigpal/jzu004

J.K. van Benthem, Conditional Probability Meets Update Logic, Journal of Logic, Language and Information 12, 409-421, 2003.

Ch. M. Grinstead and L. Snell, Introduction to Probabilities, American Mathematical Society, 1998 (second edition)

J. Hintikka and G. Sandu, 'Informational Independence as a Semantic Phenomenon', in J. E. Fenstad et al. (eds.) Logic, Methodology and Philosophy of Science, vol. 8, Amserdam: Elsevier, 571-89, 1989

A. Mann, 'A logical analysis of the Monty Hall problem', Logic Colloquium, Varna, 2010 (http://www.pps.univ-paris-diderot.fr/logic2010/contributed_talks)

Mann, A. I., G. Sandu, and M. Sevenster, Independence-Friendly Logic: A Game-Theoretic Approach. Cambridge, UK: Cambridge University Press, 2011

G. Sandu, 'Languages with imperfect information', in Modeling Strategic Reasoning, S. Gosh, R. Verbrugge and J. van Benthem (eds.), Springer, Texts in Logic and Games (forthcoming, 2015a)

G. Sandu, 'Probabilistic IF Logic', in I. Parvu, G. Sandu and I. Toader (eds.), Romanian Studies in Philosophy of Science, Boston Studies, forthcoming, (forthcoming, 2015b)

M. Sevenster, Branches of Imperfect Information: Logic, Games, and Computation. PhD Thesis, Amsterdam: University of Amsterdam, 2006.

Sevenster, M, 'Dichotomy results for Independence-Friendly prefixes of generalized quantifiers', Journal of Symbolic Logic, forthcoming, 2014

Sevenster, M. and G. Sandu, 'Equilibrium Semantics of Languages of Imperfect Information', Annals of Pure and Applied Logic 161, pp 618-31, 2010.

J. Von Neumann, Zur Theorie der Gesellschaftsspiele. Mathematische Annalen, 100, 295-320, 1928. 